\title{
Mining Discriminative Pattern to Classify the Quick and Safe Travelling in the Mode of Road Network
}

\author{
S. Sivakumar, \\ Asst.Professor in Computer Applications, \\ Thanthi Hans Roever College, Perambalur,
}

\author{
C. Chandrasekar, Ph.D \\ Associate Professor in Computer Science, \\ Periyar University, Salem,
}

\begin{abstract}
Categorization is one of the major tasks in the field of traffic network. In comparison to the other techniques which can be correlated with the categorization tries to find out all the frequent blueprints which existed in the input categorical of the traffic network satisfying the public specified least support or inequity measures like minimum confidence or information gain. Those blueprints are used shortly either as rules for rule-based classifier or training features for Support Vector Machine (SVM) categorizer. Several algorithms have been proposed to solve the categorization problem on uncertain conditions in the road transportation due to improper traffic network. In this research paper we have proposed an innovative algorithm concord which extracts discriminative patterns directly and effectively from the uncertain traffic network conditions as classified in the features which assists everyone to use the road transportation in traveling quick and safe to any destinations.
\end{abstract}

\section{General Terms}

Knowledge based, Data Mining, Pattern-based classification, road network analysis, Sequential patterns.

\section{INTRODUCTION}

Classification is one of the major tasks in the field of traffic network. In comparison to the other techniques which can be correlated with the categorization tries to find out all the frequent blueprints which existed in the input categorical of the traffic network satisfying the public specified least support or inequity measures like minimum confidence or information gain. Those blueprints are used shortly either as rules for rule-based classifier or training features for Support Vector Machine (SVM) categorizer [10]. After a characteristic variety method which usually tries to cover as many as input instances with the most discriminative patterns in various comportments. Several algorithms also have been proposed to extract the most discriminative patterns directly without expensive feature selection. As an alternative the probability distributed functions are adopted to represent the possible values and their corresponding probabilities. The uncertainty is usually caused by traffic violations, accidents and some unavoidable circumstances. Several algorithms have been proposed to solve the categorization problem on uncertain conditions in the road transportation due to improper traffic network.

In this research paper we have proposed an innovative algorithm concord which extracts discriminative patterns directly and effectively from the uncertain traffic network conditions as classified in the features which assists everyone to use the road transportation in traveling quick and safe to any destinations. In view of the fact that, these patterns which makes us to enquire the route map from the tollgates utilize the road transportation as an effectual mode for quick and safe traveling. In addition to that, it can exploit our time management effectively. The experimental results show that SVM classifier our concord algorithm outperforms about the traffic network can easily analyzed through the different methodologies for everyone to develop the road transportation as one of most important mode for us to reach any destinations as fast and secure.

\section{SEQUENTIAL PATTERNS}

All Sequential patterns indicate not only which road segments are visited, but also in which order the road segments are visited

Varying Number of Selected Features

More sequential patterns with high discriminative Power are included $>>$ high accuracy of classification

After reaches maximum >>remaining sequential Patters have limited discriminative power

Optimal number $=$ Number of selected features

Not all sequential patterns have high discriminative Power.

\section{DISTANCE CALCULATION}

Djikstra's algorithm pick the edge $\mathrm{v}$ in Fringe $(\mathrm{T})$ that has minimum distance to the starting node $\mathrm{g}(\mathrm{v})$ is minimum

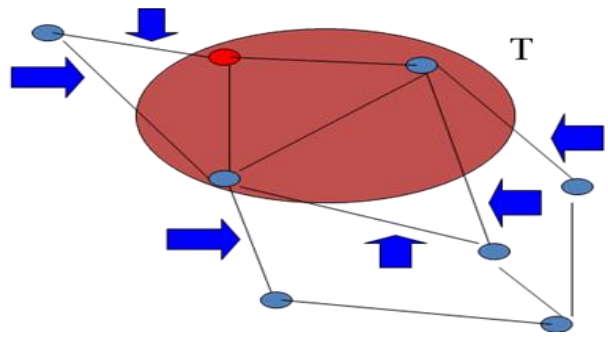

Fig 2.1: Route maps for reach the destination

Given a Graph $\mathrm{G}=(\mathrm{V}, \mathrm{E})$ and $\mathrm{T}$ a subset of $\mathrm{V}$, the fringe of $\mathrm{T}$, is defined as:

Fringe $(T)=\{(w, x)$ in $E: w \in T$ and $x \in V-T\}$

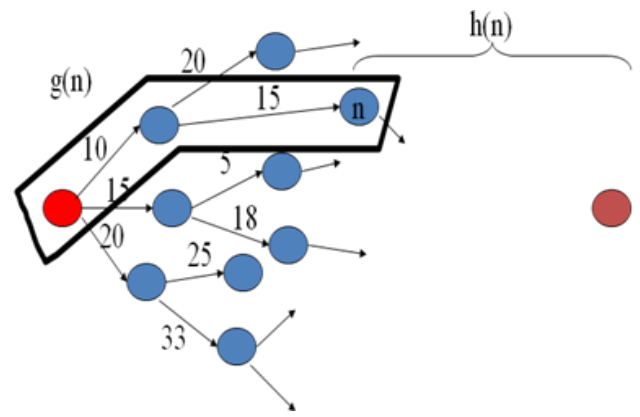

Fig 2.2: Route maps for reach the destination 
$\mathrm{f}(\mathrm{n})=\mathrm{g}(\mathrm{n})+\mathrm{h}(\mathrm{n})$

$\mathrm{g}(\mathrm{n})=$ "cost from the starting node to reach $\mathrm{n}$ "

$h(n)=$ "estimate of the cost of the cheapest path from $n$ to the goal node"

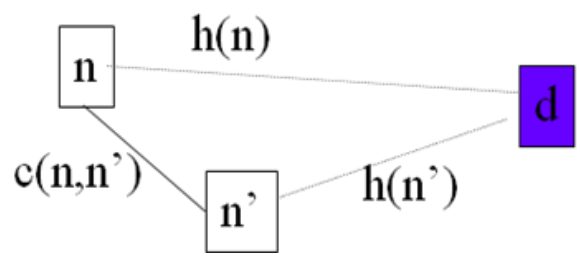

Fig 2.2.1: Node for reach the destination

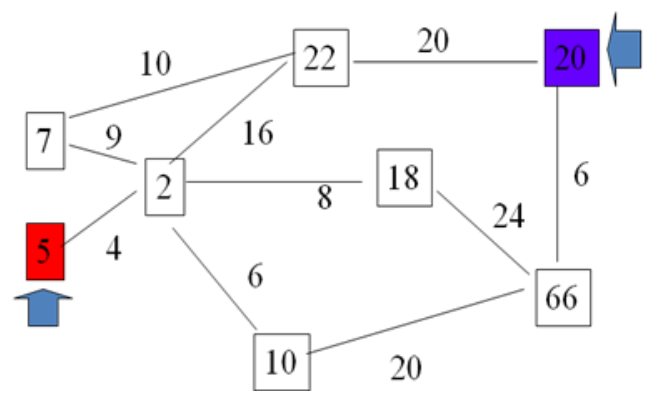

Fig 2.2.2: Example Node for reach the destination $\operatorname{dist}[\mathrm{s}] \leftarrow 0 \quad$ (distance to source vertex is zero) for all $\mathrm{v} \in \mathrm{V}-\{\mathrm{s}\}$

do $\operatorname{dist}[\mathrm{v}] \leftarrow \infty$ (set all other distances to infinity)

$S \leftarrow \varnothing \quad$ (S, the set of visited vertices is initially empty)

$\mathrm{Q} \leftarrow \mathrm{V} \quad(\mathrm{Q}$, the queue initially contains all vertices)

while $\mathrm{Q} \neq \varnothing$ (while the queue is not empty)

do $\mathrm{u} \leftarrow$ mindistance $(\mathrm{Q}$,dist) (select the element of $\mathrm{Q}$ with the min. distance)

$\mathrm{S} \leftarrow \mathrm{S} \cup\{\mathrm{u}\} \quad$ (add $\mathrm{u}$ to list of visited vertices)

for all $\mathrm{v} \in$ neighbors $[\mathrm{u}]$

do if $\operatorname{dist}[v]>\operatorname{dist}[u]+w(u, v)$ (if new shortest path found) then $\mathrm{d}[\mathrm{v}] \leftarrow \mathrm{d}[\mathrm{u}]+\mathrm{w}(\mathrm{u}, \mathrm{v})$ (set new value of shortest path)

(if desired, add traceback code)

return dist

\section{CLASSIFICATION TECHNIQUES}

Decision Tree based Methods

Rule-based Methods

Memory based reasoning

Neural Networks

Naïve Bayes and Bayesian Belief Networks

Support Vector Machine.

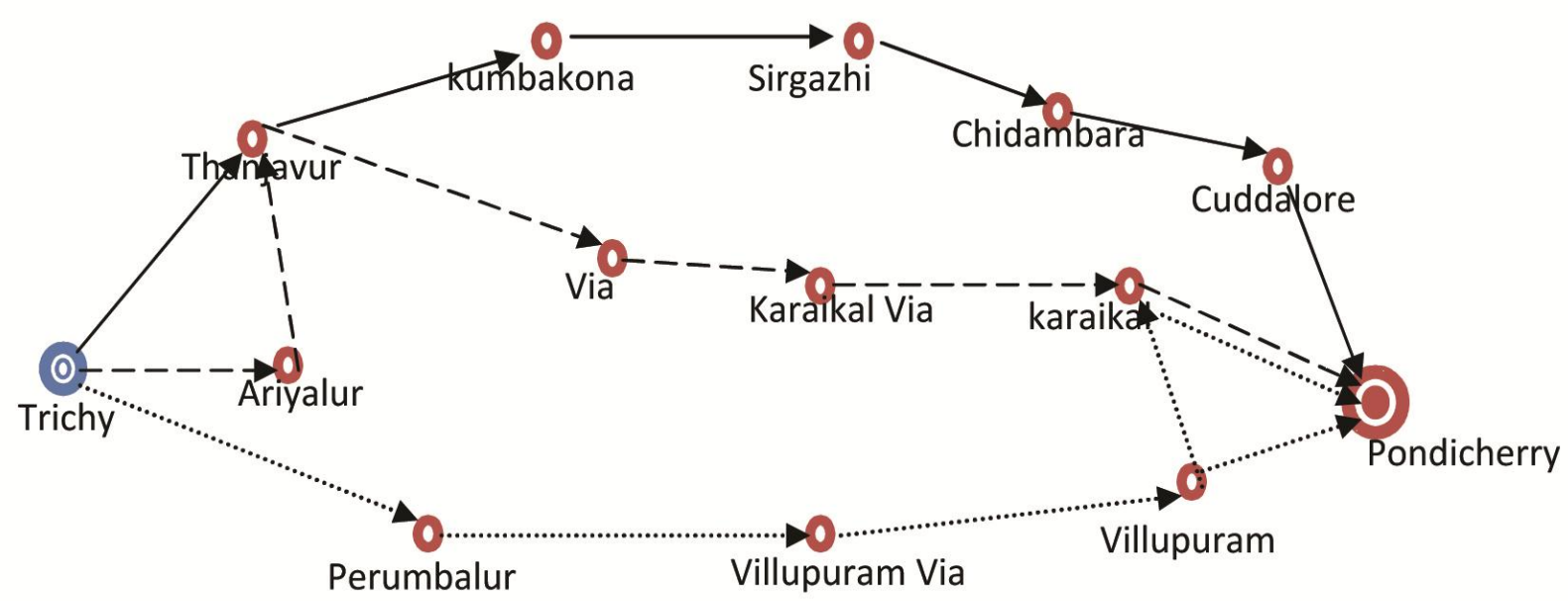

Fig 1: Possible Route maps to reach the destination

\section{PROPOSED PROCESS}

\section{Client / User Registration (the basic information)}

After the registration the server provide username and password

After login, the user fill his/her complete travel information like starting point to destination and his/her needs for the travel.
The server analysis the data (old data and current data) then produce possible three ways named $\mathrm{a}, \mathrm{b}$ and $\mathrm{c}$ to reach destination.

The user may select the route map to reach destination from the suggestions from the server or the user opts according to their needs for the travel.

When the user starts his/her travel, the server makes a note of the user's starting time and simultaneously the server provides the comprehensible information about the distance and vehicle speed chart for that particular route. According to that, 
the user can analyze the road behavior and driving methods. In addition to that, the server gives lucid details about the traffic and other issues occurred on that particular path like accidents, road repair and etc...

In case, if a trip takes an extra time in clearing the traffic and other problems, then the system automatically display the alternative routes to reach the destination and also it affords the information like speed of the vehicle, road condition, traveling hours etc.. for a safe and quick journey. If the customer (client) would not like to take the alternative path and they would like to continue their travel on that particular route signifies, then the system will demonstrate the information about hotels, entertainments, shopping malls, parks, lakes and other refreshment places for a customer to plan and enjoy on their traveling.

\subsection{PROCESS EVALUATE}

As a Result a person would like to start from the destination as Trichy and the reach point as Pondicherry. There is a possibility for multiple routes to make your travel effectively. If an individual wants to take the possible route 1 is from Trichy via Thanjavur, Kumbakonam, Sirgazhi, Chidambaram, Cuddalore to reach Pondicherry. The possible route 2 is from Trichy via Ariyalur, Thanjavur, Karaikal to reach Pondicherry. Finally the possible route 3 is from Trichy via Perambalur, Ulundurpet, Villupuram and to reach Pondicherry. From the above routes the person is potential in desiring to travel to manage the time and travel at ease.

When an individual making the comfortable sketch for travel, shopping and entertainment. Through our system it is feasible to get the information about the travel plan and route map and also it gives the comprehensible information about the road behavior. As a part of travel with the guidance of our device one can receive the travel information all the way through the SMS to their mobile phones.

Note: The server will have the complete data about the road network [1][2][3](the server collects the information from Google maps, GPS, RFID, Toll Booth and old data)

\section{CONCLUSION}

In this paper, we have presented a new mechanism for the safe and quick traveling of a customer from one source to the customer's desire destination. This system will be very effective for the people, who prefer to have safe and quick journey to their respective destinations. The server has complete collection of information about road network by using GPS and RFID technologies. The sequential discriminative pattern is analyzed by using current $\&$ old data from the server. This affords the behavior of each node to reach the destination in quick time. Here the node represents the road condition, vehicle speed, refreshment places, and peak hours.

In the proposed system, that the information is retrieved from the server and directly display to the customer by means of SMS (Short Messaging System). In future, we have a plan to change this display method to the graphical one instead of SMS.

Through my analysis and experimentation I code out the findings as pressure less travel, unwanted hassles and effective travel can be achieved from our navigation device.

Accuracy Result

The server clearly depicts the information from the GPS, Toll Booths, RFID and also compiled data. By making this process is handier for the common people.

\section{ACKNOWLEDGMENTS}

Our thanks to the experts who have contributed towards development of the work.

\section{REFERENCES}

[1] X. Y. Jin and C. H. Davis,"Multispectral IKONOS Imagery Automatic Road Extraction from HighResolution." Proceedings of IEEE International Geoscience and Remote Sensing Symposium, Vol 3. pp. 1730-1732, 2003.

[2] A. Chang S. K. Kyu and B. R. Sang, "A Road Extraction Method from Topographical Map Images." IEEE Communications, Computers and Signal Processing, Vol 2. pp.839-842, 1997.

[3] Gopalan, R., K. Kolluri, R. Batta, and M. Karwan. 1990. Modeling equity of risk in the transportation of hazardous materials. Operations Research, 38(6):961973. URL http: //links.jstor.org/sici?sici=0030-364X.

[4] Lisini, C. Tison, F. Tupin and P. Gamba, "Feature Fusion to Improve Road Network Extraction in High-Resolution SAR Images." IEEE Geoscience and Remote Sensing Letters, Vol 3, No. 2, pp. 217-221, 2006.

[5] Yan Li and Ronald Briggs, "Automatic Extraction of Roads from High Resolution Aerial and Satellite Images with Heavy Noise", World Academy of Science, Engineering and Technology, Vol. 54, pp.416-422, 2009

[6] Yu-Li Chouy H. Edwin Romeijnz Robert L. Smithx. Approximating Shortest Paths in Large-scale Networks with an Application to Intelligent. Transportation Systems. September 27, 1998

[7] Roozbeh Shad, Hamid Ebadi, Mohsen Ghods. Evaluation of Route Finding Methods in GIS Application. Dept of Geodesy and Geomatics Eng. K.N.Toosi University of Technology,

IRAN. http://www.gisdevelopment.net/technology/gis/ma03202. $\underline{\mathrm{htm}}$

[8] Thomas H. Cormen, Clifford Stein, Ronald L. Rivest and Charles E. Leiserson. Introduction to Algorithms, 2nd edition, McGraw-Hill Higher Education, 2001, ISBN: 0070131511

[9] Akgun,A.,Erkut,E.,Batta,R. (2000) On finding dissimilar paths, European Journal of Operational Research 121, 232-246

[10] C.J.C. Burges, “A Tutorial on Support Vector Machines for Pattern Recognition,"submitted to Data Mining and Knowledge Discovery, 1998. 